\title{
Why Do Certain Products Influence Grocery Store Choice? The Role of Anchor Products and Their Relationships with Other Store Choice Factors: An Abstract
}

\author{
Pilsik Choi
}

\begin{abstract}
This paper has the following two objectives: (1) to develop a concept (i.e., anchor products) that theoretically explains why some planned products are influential in store choice decisions and (2) to identify relationships between anchor products and other important store choice factors (e.g., product quality, product selection, and overall prices). To achieve the first objective, this paper proposes the concept of anchor products, drawing on anchoring effects theory and automatic cognitive processing theory. This paper also compares the concept of anchor products with other similar concepts in the store choice literature [e.g., destination categories (Briesch, Dillon, \& Fox, 2013), lead category (Chen et al., 1999), Type 1 products (Drèze \& Hoch, 1998), and category-specific store loyalty (Bell, Ho, \& Tang, 1998)] to shed light on their similarities and differences. To achieve the second objective, this paper proposes three hypotheses addressing the relationships between anchor product formation and three dominant store choice factors (product quality, product selection, and overall prices). Using a survey instrument, data were collected at two grocery stores. Based on results from logit regression and ANOVA analyses, this paper finds that favorable perceptions on (1) overall product quality and (2) product selection at a grocery store increase the likelihood that consumers choose anchor products directed at the grocery store. However, perceptions of (3) overall lower prices at a grocery store decrease the likelihood that consumers choose anchor products directed at the grocery store. The paper also offers several theoretical and managerial implications of the results.
\end{abstract}

References Available Upon Request

\author{
P. Choi $(\bowtie)$ \\ Indiana University Southeast, New Albany, IN, USA \\ e-mail: pilchoi@ius.edu
}

